\title{
Practice Spotlight: Health Outcomes Analyst in Oncology
}

\author{
Kimi Guilbert, BSc(Pharm), MSc(Pharm) \\ Health Outcomes Analyst, Epidemiology and \\ Cancer Registry, Provincial Oncology Drug \\ Program, CancerCare Manitoba \\ Assistant Clinical Professor, Faculty of Pharmacy, \\ University of Manitoba \\ Winnipeg, Manitoba
}

$\mathrm{P}$ roviding appropriate chemotherapy to patients with cancer may be expensive, and the drugs used are potentially toxic. To ensure appropriate use of health care resources, an assessment of the benefits of cancer chemotherapy is necessary and provides valuable information to both the public and funding agencies.

The Manitoba Oncology Drug Utilization and Clinical Outcomes (MODUCO) Program carries out such assessments by evaluating the therapeutic and economic outcomes of chemotherapy regimens. An initiative of CancerCare Manitoba's Provincial Oncology Drug Program (PODP), the MODUCO Program is supported by the CancerCare Manitoba/Winnipeg Regional Health Authority Oncology Pharmacotherapeutic Subcommittee. Encompassing the principles of evidence-based decision-making, stewardship, transparency, and equal access, the PODP was established in 2006 to provide the infrastructure for the effective use and financial management of oncology drugs in Manitoba.

The MODUCO Health Outcomes Analyst is a joint position funded by the PODP and the Epidemiology and Cancer Registry of CancerCare Manitoba. This position is currently filled by pharmacist Kimi Guilbert, who plays a key and unique role in the planning, organization, collection, and analysis of information relevant to determining the benefits and costs of chemotherapeutic regimens.

\section{The Analysis Process}

As new information and new drugs become available, the PODP receives requests to support the use of these new drugs or treatment regimens. In many instances, the extent, magnitude, and characteristics of the benefit to individual patients and to society are not known when a new treatment strategy is proposed. Therefore, when submissions for new therapies are presented to the CancerCare Manitoba/Winnipeg Regional Health Authority Oncology Pharmacotherapeutic Subcommittee, these gaps in the knowledge about outcomes with the proposed agent(s) must be identified.

In addition, the subcommittee recognizes the need to evaluate drug use and effectiveness after implementation of new drug regimens. Once the subcommittee has agreed to a particular evaluation project, the Health Outcomes Analyst works with key oncologists to identify the parameters necessary to evaluate the outcomes of the new therapy. At the time of writing, in early 2010, projects under way included evaluation of adjuvant use of trastuzumab in breast cancer, drug treatments in advanced colorectal cancer, use of temozolomide in glioblastoma, and use of rituximab in lymphoma and for other indications. The Health Outcomes Analyst works with a team of clinicians relevant to the therapy under evaluation and a PODP clinical pharmacist. As such, the membership of the team varies by project, depending on the treatment, target population, and delivery process. The team leaders for each project identify the data related to survival, toxic effects, and cost that are to be collected.

Once the relevant parameters for assessing the desired outcomes have been determined, the available databases are evaluated as potential sources of the information. Most important is a database of information about treatments provided to all patients in Manitoba, which contains sufficient clinical information about individual patients to allow assessment of the stage of their condition, concurrent illnesses, response to therapy, and potential therapy-induced toxic effects. This electronic health care record can be searched for individual patient characteristics or for patterns over the entire patient population. Often, some form of chart review is required to validate and complete the data required for a project. In addition, data from Manitoba Health are used where appropriate, including physician claims, hospital discharge information, and data from the Drug Program Information Network (DPIN) of Manitoba. Finally, details such as diagnosis and demographic characteristics are obtained from the Manitoba Cancer Registry. The Health Outcomes Analyst uses the information in these databases to produce a robust data repository that will be used in seeking solutions to 
targeted questions. She also assists in extracting pertinent data through a combination of chart review and electronic extraction.

Once the data have been collected, the Health Outcomes Analyst coordinates the analysis. This includes "cleaning" and validating the data, reviewing the methodology outlined in the original proposal, and performing the statistical analysis to ensure that findings are correctly interpreted. This typically involves performing survival analysis (including Kaplan-Meier curves), generating descriptive statistics, and performing Cox regression modelling. All projects are initiated with the objective of providing input to those making policy decisions.

\section{Role Requirements}

The unique role of the pharmacist as a Health Outcomes Analyst can be viewed as that of a detective: a highly skilled professional using the principles of epidemiology, statistics, and computer programming to do some super-sleuthing. Knowledge of therapeutics, clinical monitoring, and disease progression allows an understanding of the parameters that are relevant for evaluation of therapeutic outcomes. This type of work is also driven by the available data, so the pharmacist must understand the strengths and weaknesses of the data to determine the appropriate questions and corresponding methodology to obtain a valid answer.

Ms Guilbert's experience as a community pharmacist in an independent pharmacy led to her familiarity with the provincial electronic drug reimbursement database (the DPIN), which is useful in tracking treatment regimens and outcomes. Her 13 years of experience in an institutional setting provided knowledge of the institution-based drug distribution systems that are used in some analyses, as well as an understanding of the data gaps and strengths that are inherent to chart reviews and electronic data extraction with commercial charting software. A master's degree in pharmacy provided the comput- er programming, pharmacoepidemiology, and statistical skills, as well as the experience with population-based data, that are necessary for complex analysis of multiple parameters.

\section{Evolution of the Role}

The position of Health Outcomes Analyst was originally created in January 2007. Ensuring that cancer chemotherapy is being used effectively to achieve desired outcomes for patients and the Manitoba health care system justifies the expense of this pharmacist's salary. The role of the Health Outcomes Analyst and of all members of the MODUCO Program continues to evolve as the characteristics of drug regimens and the methods of care provision change with advances in drug therapy. As the members of the MODUCO Program team learn each other's skills, develop and streamline methodologies, and gain a better understanding of the potential outputs from their strategies, the role of the Health Outcomes Analyst will continue to expand. The broad combination of skills of the incumbent pharmacist will allow for her participation in a wide range of unique and satisfying roles as the program evolves.

For readers interested in the current role of the pharmacist in this outcomes assessment program, Ms Guilbert can be contacted at CancerCare Manitoba, kimi.guilbert@cancercare.mb.ca

The Practice Spotlight series highlights the accomplishments of Canadian pharmacists with unique practices in hospitals and related health care settings. If you have a unique or innovative practice, or you know someone else who should be profiled, please submit your contact information to Mary Ensom, Editor of CJHP (cjhpedit@cshp.ca), and one of our Associate Editors will be in touch with you. 